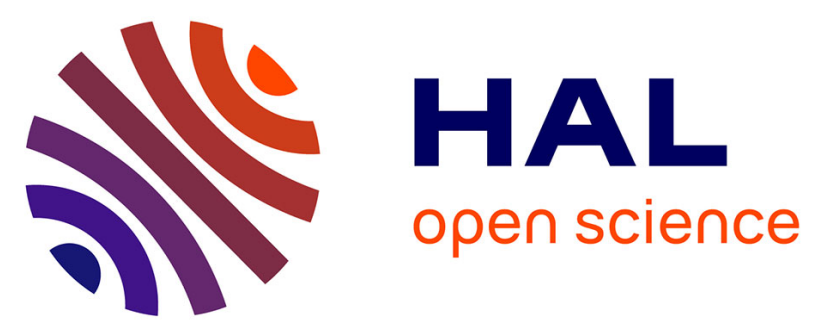

\title{
Nonsymmetrical Dynamics of the HBV Capsid Assembly and Disassembly Evidenced by Their Transient Species
}

Maelenn Chevreuil, Lauriane Lecoq, Shishan Wang, Laetitia Gargowitsch, Naïma Nhiri, Eric Jacquet, Thomas Zinn, Sonia Fieulaine, Stéphane Bressanelli, Guillaume Tresset

\section{To cite this version:}

Maelenn Chevreuil, Lauriane Lecoq, Shishan Wang, Laetitia Gargowitsch, Naïma Nhiri, et al.. Nonsymmetrical Dynamics of the HBV Capsid Assembly and Disassembly Evidenced by Their Transient Species. Journal of Physical Chemistry B, 2020, 124, pp.9987 - 9995. 10.1021/acs.jpcb.0c05024 . hal-03009814

\section{HAL Id: hal-03009814 https://hal.science/hal-03009814}

Submitted on 2 Dec 2020

HAL is a multi-disciplinary open access archive for the deposit and dissemination of scientific research documents, whether they are published or not. The documents may come from teaching and research institutions in France or abroad, or from public or private research centers.
L'archive ouverte pluridisciplinaire HAL, est destinée au dépôt et à la diffusion de documents scientifiques de niveau recherche, publiés ou non, émanant des établissements d'enseignement et de recherche français ou étrangers, des laboratoires publics ou privés. 


\section{Non-symmetrical Dynamics of the HBV}

\section{Capsid Assembly and Disassembly Evidenced}

\section{by their Transient Species}

Maelenn Chevreuil, ${ }^{* \dagger, \ddagger}$ Lauriane Lecoq, "Shishan Wang, "Laetitia Gargowitsch, ${ }^{\dagger}$

Naïma Nhiri, ${ }^{\S}$ Eric Jacquet, ${ }^{\S}$ Thomas Zinn,, Sonia Fieulaine, ${ }^{\ddagger}$ Stéphane

$$
\text { Bressanelli, }{ }^{*, \dagger} \text { and Guillaume Tresset }{ }^{*, \dagger}
$$

$\dagger$ Université Paris-Saclay, CNRS, Laboratoire de Physique des Solides, 91405 Orsay, France $\ddagger$ Université Paris-Saclay, CEA, CNRS, Institute for Integrative Biology of the Cell (I2BC), 91198, Gif-sur-Yvette, France

๑Molecular Microbiology and Structural Biochemistry (MMSB), Université Claude Bernard Lyon 1, CNRS, 7 passage du Vercors, 69367 Lyon cedex 07, France

$\S$ Université Paris-Saclay, CNRS, Institut de Chimie des Substances Naturelles, UPR 2301, 91198, Gif-sur-Yvette, France

||ESRF - The European Synchrotron, 71 avenue des Martyrs, 38043 Grenoble Cedex 9, France

E-mail: maelenn.chevreuil@laposte.net; stephane.bressanelli@i2bc.paris-saclay.fr; guillaume.tresset@universite-paris-saclay.fr 


\begin{abstract}
As with many proteins multimers studied in biophysics, the assembly and disassembly dynamical pathways of hepatitis B virus (HBV) capsid proteins are not symmetrical. Using time-resolved small-angle X-ray scattering and singular value decomposition analysis, we have investigated these processes in vitro by a rapid change of salinity or chaotropicity. Along the assembly pathway, the classical nucleation-growth mechanism is followed by a slow relaxation phase during which capsid-like transient species selforganize in accordance with the theoretical prediction that the capture of the few last subunits is slow. By contrast, the disassembly proceeds through unexpected, fractalbranched clusters of subunits that eventually vanish over a much longer timescale. On the one hand, our findings confirm and extend previous views as to the hysteresis phenomena observed and theorized in capsid formation and dissociation. On the other hand, they uncover specifics that may directly relate to the functions of HBV subunits in the viral cycle.
\end{abstract}

\title{
Graphical TOC Entry
}

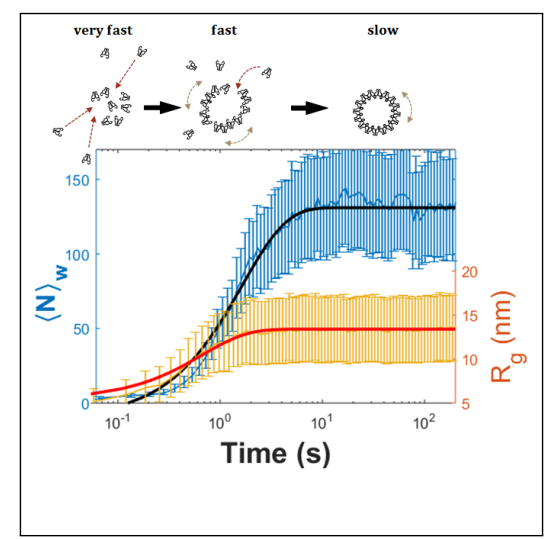

\section{Keywords}

Hepatitis B virus, self-assembly, disassembly, dynamics, TR-SAXS 


\section{Introduction}

Viruses, described as "organisms at the edge of life", do not have their own metabolism and require a host cell to replicate. All viruses comprise at least a nucleocapsid, that is, a protein shell called capsid surrounding and protecting the genome. Their survival depends on the ability of this nucleocapsid to assemble and sometimes also to completely disassemble in the host cell. Since some viral capsids ${ }^{1}$ can be easily disassembled and reassembled in vitro by change of $\mathrm{pH}$, ionic strength or temperature, in vitro studies of these mechanisms have increased in the past twenty years. However, despite these efforts, the dynamical pathway of viral assembly and disassembly is still an ill-known process.

Worldwide, about 240 million people suffer from an hepatitis B infection caused by the hepatitis B virus (HBV). It affects the liver and can cause serious complications such as cancer or cirrhosis. HBV is a DNA virus with an icosahedral capsid composed, in $95 \%$ of cases, of 120 dimers of the capsid protein, hereafter called subunits. The wild-type capsid protein is a 183-residue polypeptide comprising an assembly domain (N-terminal domain, NTD; residues 1-149) and a nucleic acid-binding domain (C-terminal domain, CTD; residues 150-183) located inside the capsid. To facilitate the study of HBV assembly and disassembly, we used a truncated protein engineered by Nassal et al. and containing only the NTD, ${ }^{2}$ which allows us to take only into account the interactions between proteins. We refer to the mutant capsid protein containing the first 149 residues as Cp149. Just like the wild-type capsid protein, Cp149 is able to make up icosahedral capsids mostly comprising 120 subunits arrayed with $T=4$ quasi-equivalence ${ }^{3}$ (see Fig. S1 in SI).

The stability of the Cp149 capsids is mainly based on a delicate balance between two interactions (see Fig. 1.A): (i) a shortrange attraction due to hydrophobic and/or van der Waals forces, which holds the subunits together; and (ii) a long-range electrostatic repulsion, which allows the capsid to disassemble. The last one, often described as one of the most important driving forces during the assembly and disassembly of the capsid, is crucially affected by the subunits charge distribution and magnitude. ${ }^{4}$ However, these data are 

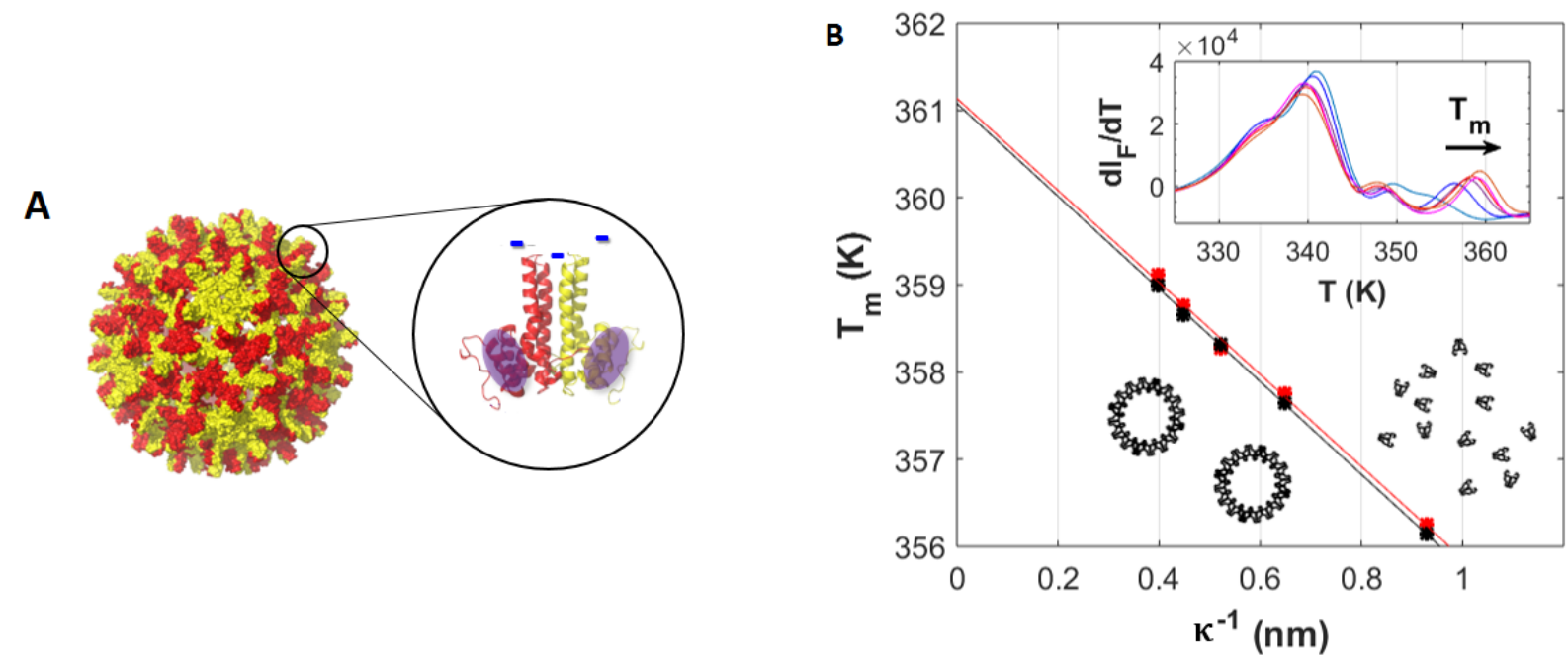

Figure 1: Thermal shift assay on empty HBV capsids. (A) A simple sub-viral particle consists of capsid proteins - associated in dimeric subunits - forming a shell and encasing the genome. The Cp149 subunits have hydrophobic sites on their sides (purple patches) and carry anionic charges (blue minus sign) on their surface. (B) Melting temperature $T_{m}$ as a function of Debye length $\kappa^{-1}$ at $\mathrm{pH} 7.5$ and different salinities. The red and black stars are experimental data replicates, and the red and black lines are linear regressions according to Eq. (1). The inset shows the derivative of the fluorescence intensity $I_{F}$ with respect to the temperature used to extract $T_{m}$ at $\mathrm{pH} 7.5$ and different salinities: from $0 \mathrm{M}$ (light blue curve) to $0.5 \mathrm{M}$ (orange curve) by steps of $0.1 \mathrm{M}$.

still unclear. Some estimated values of the corresponding energies have been deduced from theoretical calculations ${ }^{5}$ or indirectly from kinetic experiments, ${ }^{6,7}$ notably by static light scattering. ${ }^{8}$ In a previous publication, we examined the melting temperature $T_{m}$ of empty capsids derived from the cowpea chlorotic mottle virus (CCMV), a small icosahedral plant virus, the capsid of which is also made of dimeric subunits. Using a fluorescence thermal shift assay to determine $T_{m}$ and a mean-field theory, ${ }^{9}$ we were able to demonstrate a close relationship between $T_{m}$ and the subunit interactions via the effective charge of a subunit $Z$, the pairwise potential separating nearest neighbors $\epsilon$ and the Debye length $\kappa^{-1} \cdot{ }^{10}$ This relationship is given by a linear equation,

$$
T_{m}=A \epsilon+B Z^{2} \kappa^{-1}
$$

where $A$ and $B$ denote constants. The disassembly was monitored experimentally through 
the fluorescence intensity $I_{F}$ of a mixture containing capsids and fluorescent markers, whose emission was enhanced when they bound on the hydrophobic sites of the subunits. ${ }^{10}$ As the temperature was increased, the capsids experienced an order-disorder phase transition and further hydrophobic sites became exposed. $T_{m}$ was chosen as the temperature maximizing $d I_{F} / d T$.

We applied the same methodology for Cp149 capsids and Figure 1.B gives $T_{m}$ as a function of $\kappa^{-1}$, the latter being tuned by varying the salinity. By fitting the data with Eq. (1), we arrived at $\epsilon=-6 k_{B} T_{0}$ and $|Z|=1.6 e$, with $k_{B}$ the Boltzmann constant, $T_{0}$ the room temperature and $e$ the electric charge. For comparison, still in the case of Cp149 subunits, Ceres et al. estimated the subunit-subunit energy to be $-6.8 k_{B} T_{0}$ in high salt condition, ${ }^{11}$ which is close to the value we found for $\epsilon$. By contrast, the charge estimated by Sun et al. on free dimers in solution by electrophoretic mobility was about $-14.5 e$ per subunit at the same pH. ${ }^{8}$ This discrepancy can be explained by the fact that since, in our measurements, the subunits were assembled into capsids, fewer residues were ionized because less accessible to the solvent, reducing thereby the effective charge $Z$.

Sun et al. experiments were carried out on free dimers in solution by electrophoretic mobility whereas we did it on whole capsids by thermal shift assay. The difference probably comes from the charges differencies that exist between the two dimers configurations. As we said in our manuscript, the dimers organized in capsid have less ionized residues because residues are less accessible to the solvent. This ionization difference explain why the effective charge $\mathrm{Z}$ on the whole capsid is lower than on free dimers.

Despite substantial experimental and theoretical efforts devoted to the assembly and disassembly of HBV capsids, many questions remain unanswered. If the nucleation-growth mechanism ${ }^{12,13}$ is well admitted by the community for the assembly of empty capsids, the nature of the early and late intermediates is still unclear. Early intermediates for HBV capsids have been reported to be trimers ${ }^{14}$ or pentamers ${ }^{15}$ of subunits. Pierson et al. observed stable and structured late intermediates ${ }^{16}$ made of 104, 111 or 117 dimers, while Katen et al. 
as well as Kondylis et al. pointed out labile and disordered late intermediates ${ }^{17,18}$ containing defects or holes. The disassembly pathway is even more elusive, and a notable study was done by Singh and Zlotnick who combined chromatography and computer simulations to detect the presence of oligomers, the size and nature of which remain yet unknown. ${ }^{19}$

A major difficulty in the experimental study of the assembly and disassembly processes of icosahedral capsids is the coexistence of several transient species. Time-resolved small-angle X-ray scattering (TR-SAXS) with synchrotron source enables to track complex phenomena in soft matter with a temporal resolution of a few milliseconds and a spatial resolution of a few nanometers. ${ }^{20}$ Complemented with singular value decomposition (SVD), ${ }^{21,22}$ structural information and time evolution of the major long-lived species can be directly extracted with no prior assumption. A detailed description of SVD analysis is given in SI. TR-SAXS was successfully used for investigating the assembly ${ }^{23-29}$ and disassembly ${ }^{30,31}$ of a number of icosahedral capsids either empty or containing a cargo such as genomic nucleic acids, polymers or inorganic nanoparticles. Of particular interest here, Law-Hine et al. unveiled some of the transient species along the assembly and disassembly pathways of CCMV empty capsids, ${ }^{27,30}$ which are made of 90 subunits $(T=3)$. In both pathways, even though the timescales were different, one common large intermediate species comprising about 35 subunits was found. In conditions of capsid assembly, CCMV subunits have an interaction energy of $-4.6 k_{B} T_{0},{ }^{10}$ that is, weaker than that of Cp149 subunits. A question then arises: Can one still find common transient species or similar steps along the assembly and disassembly pathways of Cp149 capsids, and what would it entail in the biological context of the HBV life cycle?

Here, by combining TR-SAXS and SVD analysis, we report first on the influence of subunit concentration and salinity on the assembly pathways, and we describe several distinct phases. The disassembly pathway is then investigated and we unveil an unexpected transient species. We finish by a discussion relating our findings to the known steps of the HBV life cycle. 


\title{
Methods
}

\section{Sample preparations}

\author{
Buffer solutions
}

Table 1: Summary of the buffer solutions used in the sample preparations.

\begin{tabular}{|c|c|}
\hline BUFFER & Composition \\
\hline Lysis buffer (TN300) & $300 \mathrm{mM} \mathrm{NaCl} ; 5 \mathrm{mM}$ DTT; $50 \mathrm{mM}$ Tris $p \mathrm{H} 7.4$ \\
\hline Purification buffer & $\overline{5} \% \overline{\text { sucrose}} ; \overline{\mathrm{mM}} \overline{\mathrm{EDTA}} \overline{\mathrm{T}} \overline{\mathrm{m}} \overline{\mathrm{m}} \overline{\mathrm{D}} \overline{\mathrm{T}} \overline{\mathrm{T}} ; \overline{50} \overline{\mathrm{mM}} \overline{\mathrm{Tris}} \bar{p} \overline{\mathrm{H}} \overline{7} . \overline{5}$ \\
\hline$\overline{\text { Sucrose buffer }}$ & 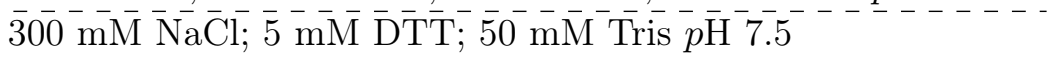 \\
\hline Purification disassembly buffer & $\overline{3} \mathrm{M}$ urea; $10 \mathrm{mM} \overline{\mathrm{DT}} ; 20 \mathrm{mM}$ Tris $p \mathrm{H} \overline{7} .5$ \\
\hline Cp $1 \overline{4} 9$ capsid buffer & $1 \overline{\mathrm{mM}} \overline{\mathrm{D}} \overline{\mathrm{T}} \overline{\mathrm{T}} ; 50 \mathrm{mM} \overline{\mathrm{CHES}} p \overline{\mathrm{H}} 9$ \\
\hline$\overline{\mathrm{C}} \mathrm{p} 14 \overline{9}$ subunit buffer & $1 \overline{\mathrm{mM}} \overline{\mathrm{DT}} \overline{\mathrm{T}} ; 50 \mathrm{mM} \overline{\mathrm{CHES}} p \overline{\mathrm{H}} 9$ \\
\hline Assembly triggering buffer & $\overline{2} \overline{\mathrm{M}} \overline{\mathrm{N}} \overline{\mathrm{Cl}} ; \overline{\mathrm{mM}} \overline{\mathrm{DT}} \overline{\mathrm{T}} ; \overline{0} \overline{\mathrm{mM}} \overline{\mathrm{C}} \overline{\mathrm{H}} \overline{\mathrm{ES}} \bar{p} \overline{\mathrm{H}} \overline{9}$ \\
\hline Disassembly triggering buffer & $\overline{6} \overline{\mathrm{M}}$ urea; $\overline{1} \mathrm{mM} \overline{\mathrm{DTT}} ; \overline{50} \mathrm{mM} \overline{\mathrm{CH}} \overline{\mathrm{ES}} \overline{\mathrm{H}} \overline{9}$ \\
\hline
\end{tabular}

\section{Expression of Cp149 capsids}

The expression of Cp149 capsids was adapted from a protocol developed previously. ${ }^{32}$ Briefly, the pRSF-T7-HBc149opt plasmid was incorporated into E.coli BL21*CodonPlus cells and grown at $37^{\circ} \mathrm{C}$ overnight on a LB-agar plate containing $50 \mu \mathrm{g} . \mathrm{mL}^{-1}$ kanamycine and 34 $\mu \mathrm{g} . \mathrm{mL}^{-1}$ chloramphenicol. Then, a single colony was inoculated into $2 \times 10 \mathrm{~mL} \mathrm{LB}$ medium and grown at $37^{\circ} \mathrm{C}$ overnight. It was then transferred into $2 \times 500 \mathrm{~mL}$ of LB medium containing $50 \mu \mathrm{g} \cdot \mathrm{mL}^{-1}$ kanamycine and $34 \mu \mathrm{g} \cdot \mathrm{mL}^{-1}$ chloramphenicol and incubated for 5 to 6 h at $37^{\circ} \mathrm{C}$. When $\mathrm{OD}_{600}$ reached 0.8 , the bacterial cells were grown at $25^{\circ} \mathrm{C}$ overnight after induction with $1 \mathrm{mM}$ IPTG.

\section{Purification of Cp149 capsids}

The purification of Cp149 capsids was adapted from a protocol developed previously. ${ }^{32}$ Bacterial cells containing Cp149 capsids were pelleted by centrifugation (6,000×g, 20 min) and re-suspended in $15 \mathrm{~mL}$ of lysis buffer. $15 \mathrm{mg}$ of chicken lysozyme, $300 \mu \mathrm{L}$ of protease 
inhibitor cocktail solution $50 \times$ and $750 \mu \mathrm{L}$ of $10 \%$ Triton-X-100 were added to the cell suspension and incubated on ice for $45 \mathrm{~min}$. Then, benzonase nuclease was added to the suspension and mixed for $30 \mathrm{~min}$ at room temperature. The cells were lysed by sonication and then centrifuged at $8,000 \times \mathrm{g}$ for $1 \mathrm{~h}$ to remove cell debris. The supernatant was loaded onto a step gradient from 10 to $60 \%$ (w/v) sucrose buffer and centrifuged with a SW-32Ti Beckman Coulter rotor at $28,800 \times \mathrm{g}$ for $3 \mathrm{~h}$ at $4^{\circ} \mathrm{C}$. The gradient fractions containing Cp149 capsids were identified by $15 \%$ SDS-PAGE and precipitated by slow addition of $\mathrm{AmSO}_{4}$ to $20 \%$ saturation. After incubation on ice for $1 \mathrm{~h}$ and centrifugation at $25,000 \times \mathrm{g}$ for $1 \mathrm{~h}$, pellets were resuspended in purification buffer. The protein solution was centrifuged again for 15 min to remove insoluble pellets and the supernatant containing soluble capsids was dialyzed overnight in purification buffer at $4^{\circ} \mathrm{C}$. For disassembly experiments, the capsid solution was dialysed against capsid buffer. The capsid stock concentration, with $29450 \mathrm{M}^{-1} . \mathrm{cm}^{-1}$ as molar attenuation coefficient, was $2.4 \mathrm{mg} \cdot \mathrm{mL}^{-1}$ and the absorbance ratio $\mathrm{A}_{260} / \mathrm{A}_{280}$ was around 0.61 .

\section{Purification of Cp149 subunits}

All Cp149 subunits used for assembly assays were dissociated from previously purified Cp149 capsids into dimeric subunits as described elsewhere. ${ }^{33}$ Briefly, Cp149 capsids expressed and purified from E.coli were disassembled by dialysis at $4^{\circ} \mathrm{C}$ into disassembly buffer. Following dialysis, the solution was centrifuged at $20,000 \times \mathrm{g}$ for $15 \mathrm{~min}$ at $4^{\circ} \mathrm{C}$ in order to remove all the elements that could aggregate and then obstruct the separation column. Cp149 subunits were then purified by size exclusion chromatography (SEC) using a Superdex S200 increase GL previously equilibrated in disassembly buffer. Fractions containing subunits were identified with absorbance at $280 \mathrm{~nm}$ and dialysed against subunit buffer. The subunit stock concentration was $1.17 \mathrm{mg} . \mathrm{mL}^{-1}$ and the absorbance ratio $\mathrm{A}_{260} / \mathrm{A}_{280}$ was around 0.57. 


\section{Small-angle X-ray scattering}

\section{Assembly experiments}

Time-resolved small-angle X-ray scattering (TR-SAXS) assembly experiments were carried out with the ID02 beamline at the European Synchrotron Radiation Facility (Grenoble, France). ${ }^{34}$ SAXS patterns were recorded using a high speed and ultra-low noise area CCD detector (Rayonix MX-170HS). The sample-to-detector distance was set to $1.3 \mathrm{~m}$ or $3 \mathrm{~m}$. With scattering wavenumbers $q=4 \pi / \lambda \sin (\theta / 2)$, where $\theta$ is the scattering angle and $\lambda=$

$0.995 \AA$ the X-ray wavelength (at a photon energy of $12.46 \mathrm{keV}$ ), these distances provided $q$ values ranging from $6.4 \times 10^{-2}$ to $5.7 \mathrm{~nm}^{-1}$ or from $2.7 \times 10^{-2}$ to $2.4 \mathrm{~nm}^{-1}$. The experimental setup included a stopped-flow device (Biologic SFM-400) used to mix the subunits with the assembly triggering buffer. The mixed solutions were then injected through a quartz capillary of diameter $1.4 \mathrm{~mm}$ and the flow was stabilized with a hard stop valve. The total delay time for the solution to reach the capillary including the mixing time was less than $10 \mathrm{~ms}$ in all experiments. Beam exposure time was set to $20 \mathrm{~ms}$. The scattering intensities were converted into absolute units after subtracting the contribution of the buffer solution. Experimental uncertainties were calculated after circular averaging using the SAXSutilities software package.

\section{Disassembly experiments}

TR-SAXS disassembly experiments were carried out with the SWING beamline at the Synchrotron SOLEIL (Gif-sur-Yvette, France). Two-dimensional scattering images were recorded with an AVIEX large-area CCD detector. The sample-to-detector distances was set to $1.5 \mathrm{~m}$ which provided scattering wavenumbers $q$ ranging from about $5 \times 10^{-2}$ to $2.5 \mathrm{~nm}^{-1}$. The Cp149 capsids were manually mixed with the disassembly triggering buffer. The mixed solution was then injected via an injection robot through a capillary. The total delay time including mixing, safety checks and injection was a few minutes, which explains why we did 
not capture the early stage of the kinetics. Beam exposure time was set to $250 \mathrm{~ms}$. The scattering intensities were also converted into absolute units after subtracting the contribution of the buffer solution. The experimental uncertainties were calculated after circular averaging using the FOXTROT package.

\section{Results}

\section{Assembly experiments}

In vitro $\mathrm{HBV}$ assembly experiments were performed by quickly mixing a solution of subunits with a high salt solution, both at $\mathrm{pH} 9$ (See detailed description of sample measurements in Methods.). The temperature was maintained at $\mathrm{T}=20^{\circ} \mathrm{C}$ and the final concentrations in subunits and salt are reported in the table 2. A total of six different conditions were assayed. The assembly kinetics were performed and collected over different time intervals, ranging from 6 minutes for the shortest to 19 minutes for the longest.

Table 2: The asterisks indicate conditions with several replicates. SUMMARY TABLE OF THE EXPERIMENTAL CONDITIONS

\begin{tabular}{|c|c|c|c|c|}
\hline Cp149 subunit concentration (g.L ${ }^{-1}$ ) & 0. & & 0.6 & 0.9 \\
\hline $\mathrm{NaCl}$ concentration $(\mathrm{M})$ & $0.5^{*} 1^{*}$ & $1.5^{*}$ & $0.5 \mid 1^{*}$ & 0.5 \\
\hline
\end{tabular}

SAXS curves from a typical assembly kinetics are presented on Fig. 2.A. In the early time steps, the scattering intensities exhibited a monotonic decrease with the wavenumber $q$ indicating that subunits were the only species in solution. A few seconds after triggering the assembly, the scattering intensities displayed oscillations with a first minimum at $q=0.23$ $\mathrm{nm}^{-1}$, close to the $q$-value expected for a $T=4$ capsid, which indicates the formation of capsid-like objects where HBV forms $T=3$ were in the minority (see Fig. S2 in SI for the SAXS pattern of subunits and capsids). A SVD analysis was then performed on the matrix of scattering intensities $\mathbf{I}$ and revealed that each set of data could be approximated by a matrix of rank 2 (see Fig. S4 in SI). 
It allowed us to reconstruct the time-resolved scattering intensities through a non-linear least-squares curve fitting by considering only two species coexisting in solution, i.e., $T=4$ capsids and free subunits, and therefore, to estimate the evolution of the mass fractions. A typical reconstruction is presented on Fig. 2.B, and the final mass fraction in capsids was estimated to be around 20\%. All the final mass fractions are summarized in Fig. 2.C. One can see first that there are a critical subunit concentration and a critical salinity to allow the formation of capsids. Secondly, the final mass fraction in capsids is a function of the initial subunit and salt concentrations. Both observations are consistent with previous experiments $^{11}$ as well as theoretical models based on the law of mass action and assuming the presence of only free subunits and capsids. ${ }^{35}$
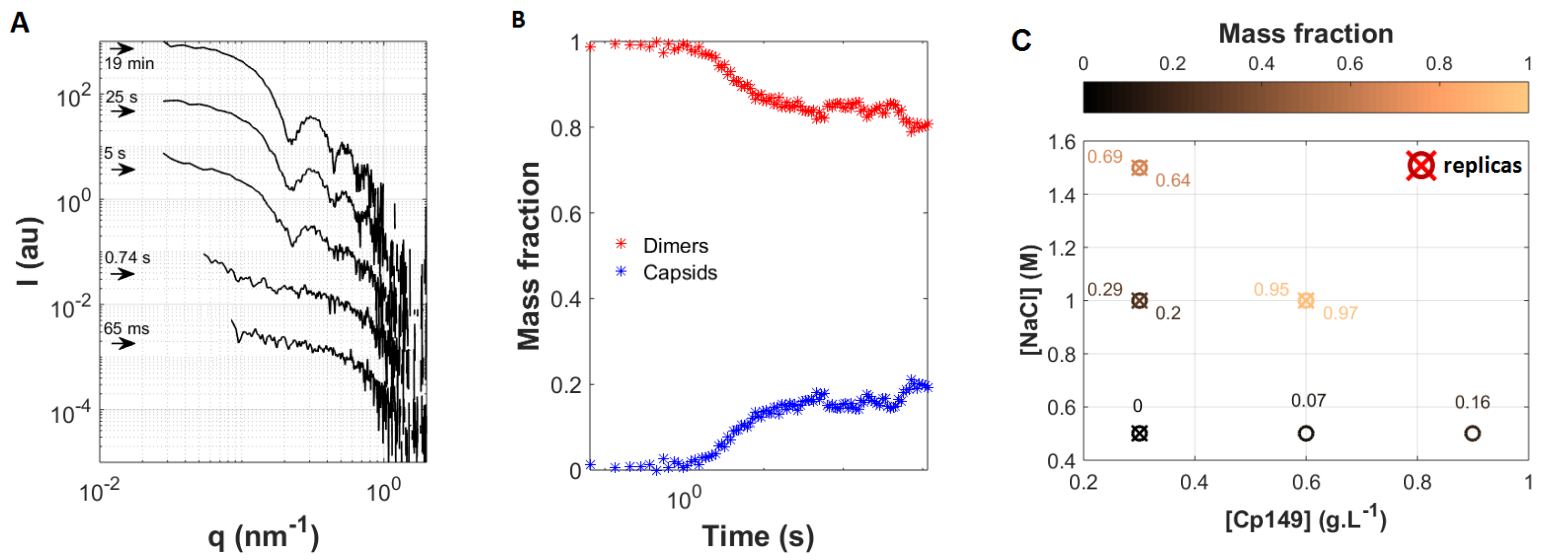

Figure 2: TR-SAXS data for the assembly of Cp149 empty capsids. (A) TR-SAXS patterns obtained at pH 9 and 0.9 g.L. $\mathrm{L}^{-1}$ of subunits. The assembly was triggered with $0.5 \mathrm{M}$ of salt. The patterns are shifted for clarity. (B) Mass fractions of subunits (red) and capsids (blue) as a function of time. (C) Summary graph of the final mass fractions in capsids as a function of salt and subunit concentrations.

In order to get a better insight into the assembly pathways, we extracted the radius of gyration $R_{g}$ as well as the mean aggregation number $\langle N\rangle_{w}$ at each time step and for each kinetic experiment by using the code DATRG. ${ }^{36}$ We subsequently computed the corresponding timescales, i.e., $\tau_{R_{g}}$ and $\tau_{\langle N\rangle_{w}}$, by fitting the latter quantities with single exponential decay functions (see Table S1 in SI). An example of the extracted time evolutions for $\langle N\rangle_{w}$ and $R_{g}$ as well as their respective fits are presented in Fig. 3.A. 
A

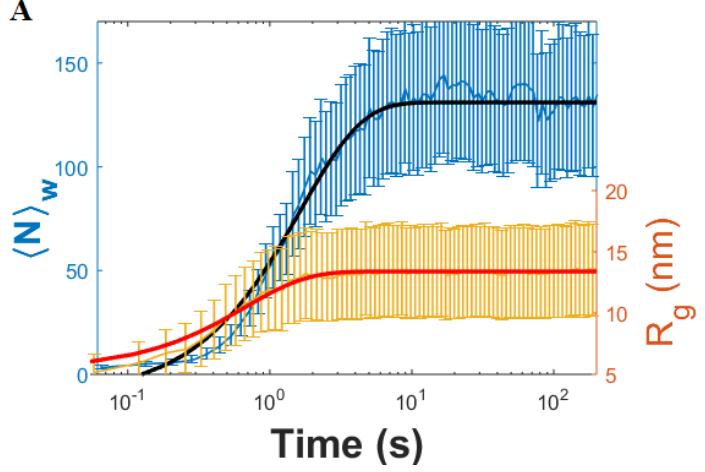

C

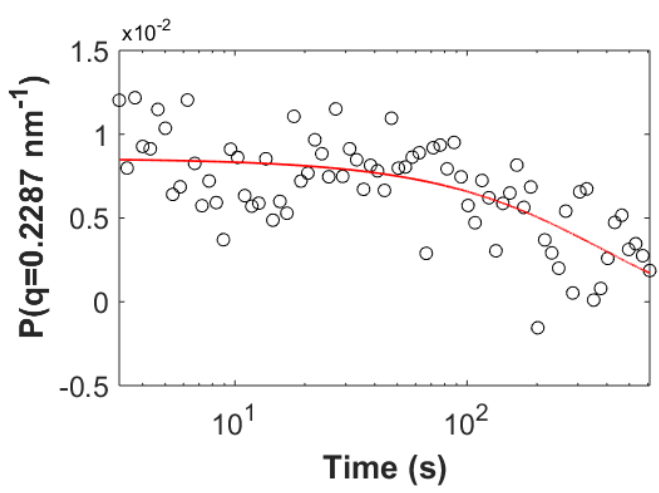

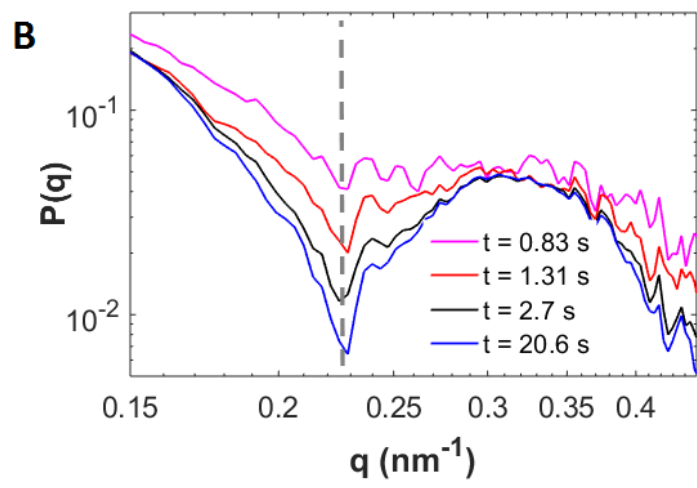
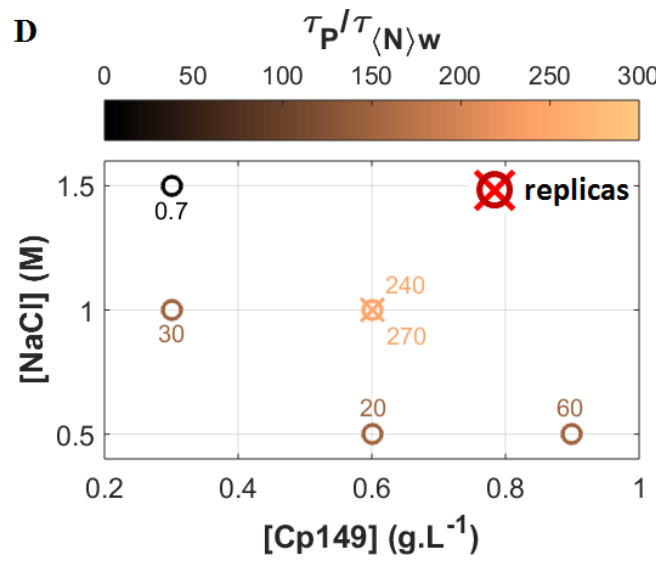

Figure 3: Assembly phases. (A) Evolution of $\langle N\rangle_{w}$ (blue) and $R_{g}$ (orange) as a function of time. The black and red curves show fits with a single exponential decay function. (B) Form factors $P(q)$ at different time points. The gray dashed line indicates $q=0.2287 \mathrm{~nm}^{-1}$. (C) Values of the form factors at $q=0.2287 \mathrm{~nm}^{-1}$ versus time. The red line is a single exponential decay fit yielding an estimate for the structural relaxation time $\tau_{P}$. (D) Summary graph of the ratios of the extracted characteristic timescales: $\tau_{P} / \tau_{\langle N\rangle_{w}}$.

The extracted timescales $\tau_{\langle N\rangle_{w}}$ and $\tau_{R_{g}}$ revealed two early phases during the assembly process. As shown on Fig. 3.A, there was a first phase, ascribed to a nucleation, where both the size and the mass of the species increased but at different rates, $R_{g}$ plateauing earlier than $\langle N\rangle_{w}$. This nucleation phase was followed by a growth phase, where $\langle N\rangle_{w}$ continued to increase while $R_{g}$ no longer evolved significantly. After a few seconds, $R_{g}$ and $\langle N\rangle_{w}$ both reached their equilibrium values. However, if $\langle N\rangle_{w}$ and $R_{g}$ remained stable as early as a few seconds after the onset of assembly, we still noticed form factors arches more pronounced over time (Fig. 3.B), which reflects self-organization process dynamics. This is highlighted on Fig. 3.C that depicts the time evolution of the first minimum of the form factors $P(q)$, and which allowed us to extract a third timescale $\tau_{P}$. For the kinetics presented on Fig. 3.C, $\tau_{P}$ 
was around $400 \pm 70 \mathrm{~s}$, which is two orders of magnitude larger than the two other timescales. For the other kinetics, $\tau_{P}$ was between $303 \pm 65 \mathrm{~s}$ and $588 \pm 70 \mathrm{~s}$. This evolution can be ascribed to a third phase, namely, the relaxation of capsid-like objects into fully assembled capsids.

By comparing $\tau_{\langle N\rangle_{w}}$ and $\tau_{P}$ across the various kinetics (Fig. 3.D), we identified a different assembly dynamics depending upon the experimental conditions. The kinetics carried out at 0.3 g. $\mathrm{L}^{-1}$ in subunits and $1.5 \mathrm{M}$ in salt exhibited values for $\tau_{\langle N\rangle_{w}}$ and $\tau_{P}$ with the same order of magnitude, unlike all the other kinetics. Additionally, the ratio $\tau_{\langle N\rangle_{w}} / \tau_{R_{g}}$ was five to ten fold higher than the ratios calculated in the other experimental conditions (see Fig. S7 in SI). These observations indicate that the relaxation phase did not occur under high salt and low subunit concentration.

To summarize on the assembly pathways, our findings suggest that, during the nucleation phase, several subunits quickly agglomerated to form disordered objects of the size of a capsid but with a lower mass. During the growth phase, the objects self-organized while capturing free subunits in solution until reaching a mass close to that of a capsid. At this point, these capsid-like objects continued to self-organize and relaxed structurally towards icosahedral capsids. However, in harsh conditions, i.e., with high salt and low subunit concentration, the latter phase was inhibited. We believe that the electrostatic repulsion between subunits was screened by the highly concentrated salt, which subsequently enhanced the effective subunitsubunit attraction. As a result, the capsid-like objects could not rearrange themselves and ended up kinetically trapped with many defects.

\section{Disassembly experiments}

In vitro disassembly of Cp149 capsids was carried out by mixing manually a solution of capsids with a $6 \mathrm{M}$ urea solution, both at $\mathrm{pH} 9$ and $10^{\circ} \mathrm{C}$ (See detailed description of sample measurements in Methods.). The sample was dialyzed in capsid buffer overnight two days before the measurements. 

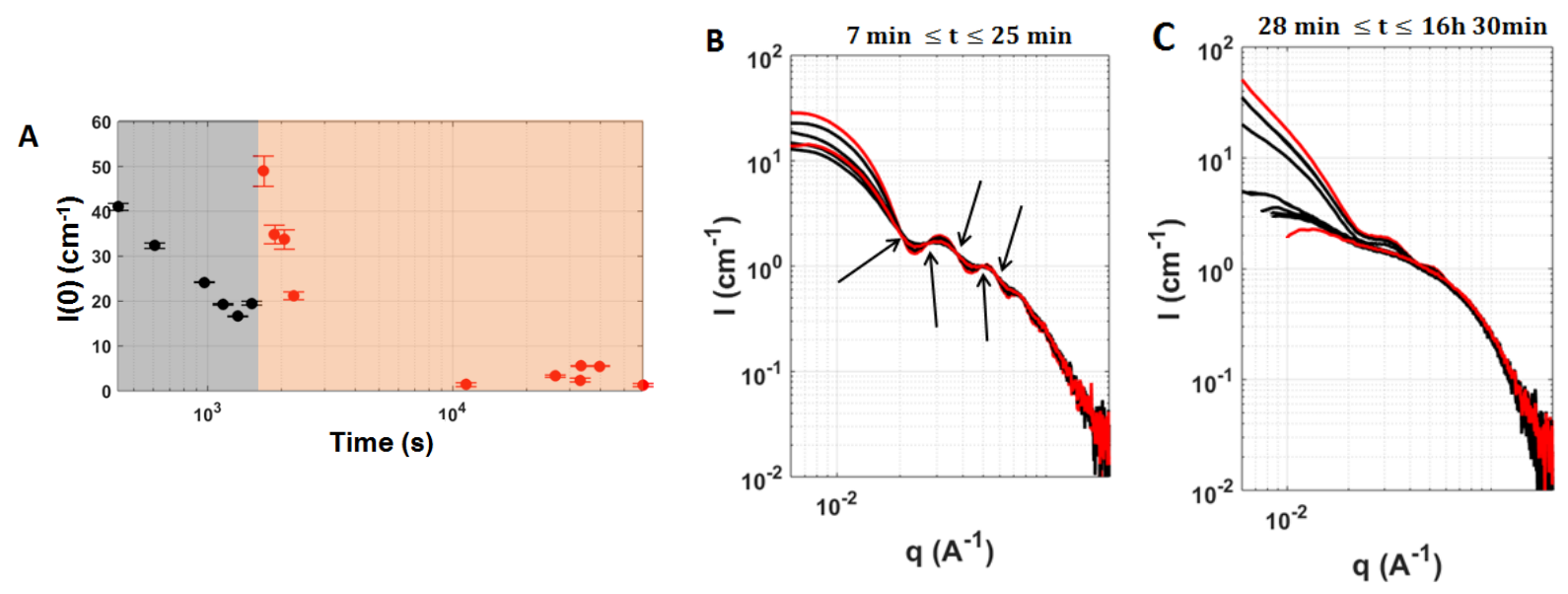

Figure 4: TR-SAXS data for the disassembly of Cp149 capsids. The final subunit and urea concentrations were 2.4 g. $\mathrm{L}^{-1}$ and $3 \mathrm{M}$, respectively. (A) Time evolution of the forward scattering intensity $I(0)$ from $7 \mathrm{~min}$ to $16 \mathrm{~h} 30$. The gray and red colored areas delimit the two phases of the disassembly. (b,c) Superimposed scattering patterns collected at early and late time steps. The patterns in red are the first and the last within each time range. The arrows in (B) point out isoscattering point.

Figure 4.A depicts the time evolution of $I(0)$, estimated by using the code DATRG, for the disassembly experiment. Two phases can be clearly seen: (i) An early short phase, up to $25 \mathrm{~min}$ (gray area), where the forward scattering intensities $I(0)$ decreased over time; and (ii) a second longer phase (red area) with a rapid rise of $I(0)$ at the beginning of the phase followed by a slow decrease to an equilibrium state. The superimposed patterns at the early stage of the kinetics are shown on Fig. 4.B and exhibited isoscattering (or isobestic) point (see black arrows), which suggested the presence of only two coexisting species in solution over this time range. The superimposed patterns at the late stage of the kinetics showed a rapid rise of the intensities at the beginning (Fig. 4.C). That rise of scattering intensity visible at low wavenumbers led to values exceeding those obtained initially with pure capsids, which suggested the formation of larger-than-capsid clusters.

In order to estimate the number of contributing species during the early phase, we performed a SVD analysis on the time-resolved scattering patterns. Solely two species were contributing significantly and the comparison of the scattering patterns extracted from the SVD analysis with the experimental patterns of capsids and subunits showed a good agree- 
ment (data not shown). As far as the whole kinetics is concerned, SVD analysis indicated three significant contributions (see Fig. S5 in SI), namely, free subunits, capsids and an unknown transient species, and Fig. 5.A shows their extracted scattering patterns. Notice that the Guinier region on the pattern of the transient species (in magenta) was not visible in our range of $q$-values, which confirmed that the species was much larger than a capsid.
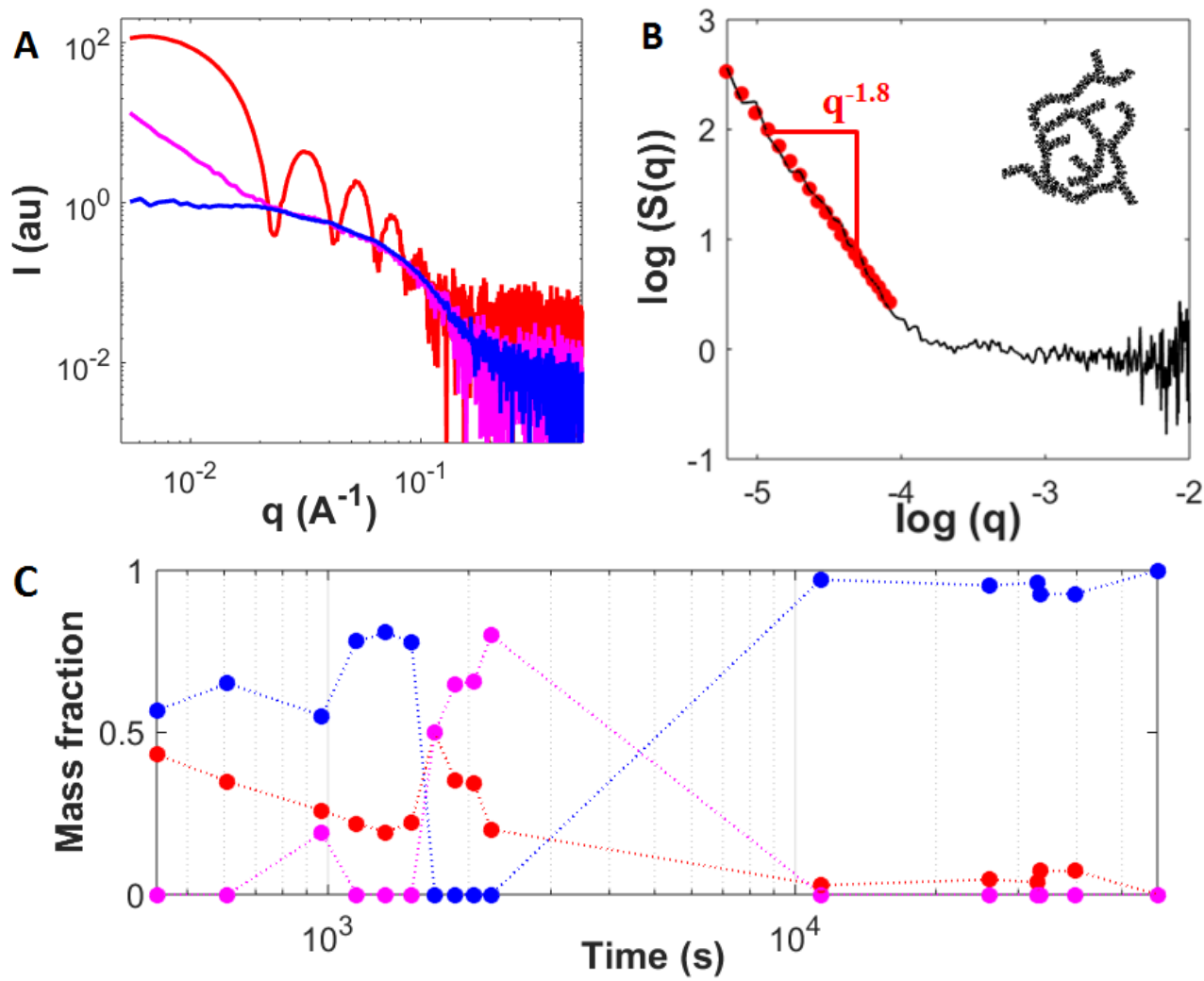

Figure 5: Species involved in the disassembly. (A) Scattering patterns of the three species in solution: subunits in blue, clusters in pink and capsids in red. (B) Structure factor $S(q)$ of the clusters. The schematics represents the branching fractal structure. (C) Mass fractions of the three species as a function of time estimated by a least-squares minimization.

We then tried to understand the nature of the clusters. We first repeated the disassembly experiment by SAXS and static light scattering (SLS) in various conditions, and each time, we observed a strong but transient increase of the scattering intensity (see Fig. S9 in SI). Consequently, we could rule out the possibility of radiation damage or of an experimental mistake. Then, we noticed the almost perfect overlap between the scattering patterns of subunits and that of the clusters at high $q$-values, which suggested that the latter were made 
of subunits. Using the small and medium $q$-values of the scattering curve, we looked for information about their long-range organization. Indeed, this region of the curve reflects the fractal nature of the clusters (see Fig. S10 in SI). As shown on Fig. 5.B, by dividing the form factor of subunits, we computed the structure factor $S(q)$ of the clusters. We thus obtained a fractal dimension of 1.8, indicating that inside the clusters, more or less attractive and repulsive interactions counterbalance each other, which is reminiscent to the self-avoiding walk model in polymers. As a consequence, one can hypothesize that the oligomers observed by Singh et al. ${ }^{19}$ whose size and nature remained unknown, were clusters of subunits organized into a branching fractal structure. Figure 5.C gives the estimated mass fraction of each species via a least-squares minimization. It should be noted that there was a significant amount of subunits (approximately 60\%) from the beginning of the kinetics, which can be explained by the fact that the first data point was collected seven minutes after the mixing due to technical constraints, and also because the capsids were stored at $\mathrm{pH} 9$, which may have contributed to start the dissociation process.

\section{Discussion \& Conclusions}

In the literature, for HBV and many other viruses, the assembly dynamics of empty capsids is often described by the nucleation-growth mechanism. If our observations do not question this model, they introduce a third slow phase of relaxation. It is not yet clear whether the relaxation phase reflects the self-organization of capsid-like objects into icosahedral capsids made of 120 subunits rather than the capture of the last missing subunits. It seems quite plausible that both events occur simultaneously, in particular because they are not mutually exclusive. Indeed, even if $\langle N\rangle_{w}$ seems unchanged during the relaxation phase, given the large uncertainties shown on Fig. 3.A, it is possible that we were not able to detect the capture of a few missing subunits. This assumption of a slow relaxation following the growth phase is supported by theoretical studies, ${ }^{13}$ which demonstrated that the insertion of the final 
subunit is energetically costly but ensures the metastability of capsids against a change of the environment. It is also supported by previous studies on Cp149 capsids $^{16,18}$ reporting the presence of long-lived species made of 104, 111 or 117 subunits in the late stage of assembly kinetics. Besides, one can imagine that such incomplete capsids may also display a number of defects in their icosahedral order. In a recent article, Panahandeh et al. showed through Monte Carlo simulations that a disorder-order transition can take place during the assembly of icosahedral capsids. ${ }^{37}$ The transition allow the objects to correct the defects in the icosahedral order, such as rearrangement of pentamers or hexamers of dimers positioned in a "wrong" location, that inevitably occur during the growth phase. Depending upon the elastic and subunit-subunit interaction energies, the authors observed the formation of transient disordered capsid-like objects that can evolve either with icosahedral order or into misassembled shells. We think therefore that the relaxation phase is closely related to the disorder-order transition, with capsid-like objects correcting their structural defects while capturing the last missing subunits. High salt conditions most likely induce high elastic and interaction energies impairing the correction of misplaced subunits, and lead to the kinetically trapped, disordered capsid-like objects seen in our experiments.

As exposed earlier, we did not find, in our assembly conditions, one of the late intermediates comprising more than 100 subunits previously proposed in the literature. ${ }^{16,18}$ It should be noted that TR-SAXS cannot distinguish reliably objects with similar form factors. ${ }^{22}$ In particular, the late intermediates postulated in nucleation-growth-closure models have form factors too close to that of a complete capsid (see Fig. S8 in SI) to be separated by SVD. Perhaps for similar reasons, we could not distinguish clearly $T=3$ capsids from $T=4$. Sun et al. ${ }^{38}$ found a significant change in the proportions of $T=3$ and $T=4$ capsids upon an increase of salt concentration. This result is also reported in numerous publications of Zlotnick, Jacobson and coworkers (e.g. ${ }^{39}$ ) using resistive-pulse sensing, a single-molecule technique performed at much lower protein concentrations than the present work. In a recent TR-SAXS study much more similar to ours, ${ }^{40}$ Asor et al. obtained very similar results to 
ours but did find in their highest salt condition significant amounts of $T=3$ capsids, which they estimated to plateau at $20 \%$ of the mass fraction. Unlike them, the position of the first minimum on our scattering patterns in varying salt conditions, which allows estimation of the $T=3$ to $T=4$ capsids ratio, remained remarkably fixed (see Table S2 in SI), close to the $q$-value expected for a $T=4$ capsid. This first minimum is also close to the one expected for a solution with $20 \%$ (mass fraction) of $T=3$ capsids (see Table S2 in SI), therefore we cannot rule out the presence of a small proportion of $T=3$ capsids. However, unlike Asor et al. in a single experiment, ${ }^{40}$ all our SVD analyses indicated an effective rank of 2 in all our experiments. These discrepancies, particularly the negligible variation of the fraction of $T=3$ capsids in our data sets, may be due to the fact that the experiments of Asor et al. ${ }^{40}$ were always performed below $0.5 \mathrm{M}$ of ammonium acetate while ours were performed with $0.5 \mathrm{M}$ of $\mathrm{NaCl}$ or more. Nevertheless, our assembly studies in accordance with recent work, ${ }^{41}$ emphasized the rapid formation (in a few seconds) of capsid-like objects through a classical nucleation-growth mechanism in two phases, which could allow an almost instantaneous protection of the genome in vivo. These phases are then followed by a slow structural relaxation (in several minutes) of the capsid-like objects into icosahedral capsids while adding the few missing subunits. In vivo, the latter phase may allow the capsid to strengthen, providing thus an efficient and solid protection for the genome.

We were also able to confirm previous observations about the non-symmetrical dynamics of the assembly and disassembly of HBV empty capsids. ${ }^{19}$ Unlike other viruses such as CCMV for which the disassembly pathway of empty capsids ${ }^{30}$ is almost a mirror image of the assembly pathway, ${ }^{27}$ the assembly of HBV empty capsids occurs quickly - over ten minuteswhile the disassembly requires more than ten hours, even in harsh non-physiological conditions (Fig. 6). In the case of an RNA virus like CCMV, the disassembly of the capsid is biologically relevant because the RNA has to be released into the cytoplasm of the infected cell. This is not the case with HBV for which there is no dissociation per se: Capsids loaded with viral DNA partially dissociate when transferred into the nucleus, but proteins remain 
partly associated with the genomic DNA (see Fig. S11 in SI). Despite the non-physiological conditions of our experiments, i.e., $3 \mathrm{M}$ of urea at $\mathrm{pH} 9$, the long and tough in vitro disassembly process is in adequacy with what happens in vivo. Indeed, this behaviour must be related to the resilience of the subunits that allow them to remain associated with genomic DNA, and consequently, harsh conditions are necessary to completely dissociate the capsid in vitro. Moreover, the capsid has to be robust enough to host partially double-stranded DNA during the HBV life cycle (see Fig. S11 in SI). There must be strong interactions between subunits and it explains why it is so difficult to completely dissociate them without the right cellular factors. It is also quite remarkable that in these conditions the process goes through a transient phase in which larger-than-capsid branching fractal clusters made of subunits form. This phenomenon is unusual with proteins under an X-ray beam, where aggregation is normally irreversible and due to radiation damage. Here it could be due to a change in protein conformation caused by urea, which first leads to form aggregates before the entropy finally plays its role by disassembling these into free subunits. These branching fractal clusters could reflect the ability of HBV subunits to self-organize in extended patterns suitable for their association with the genomic DNA in the nucleus.

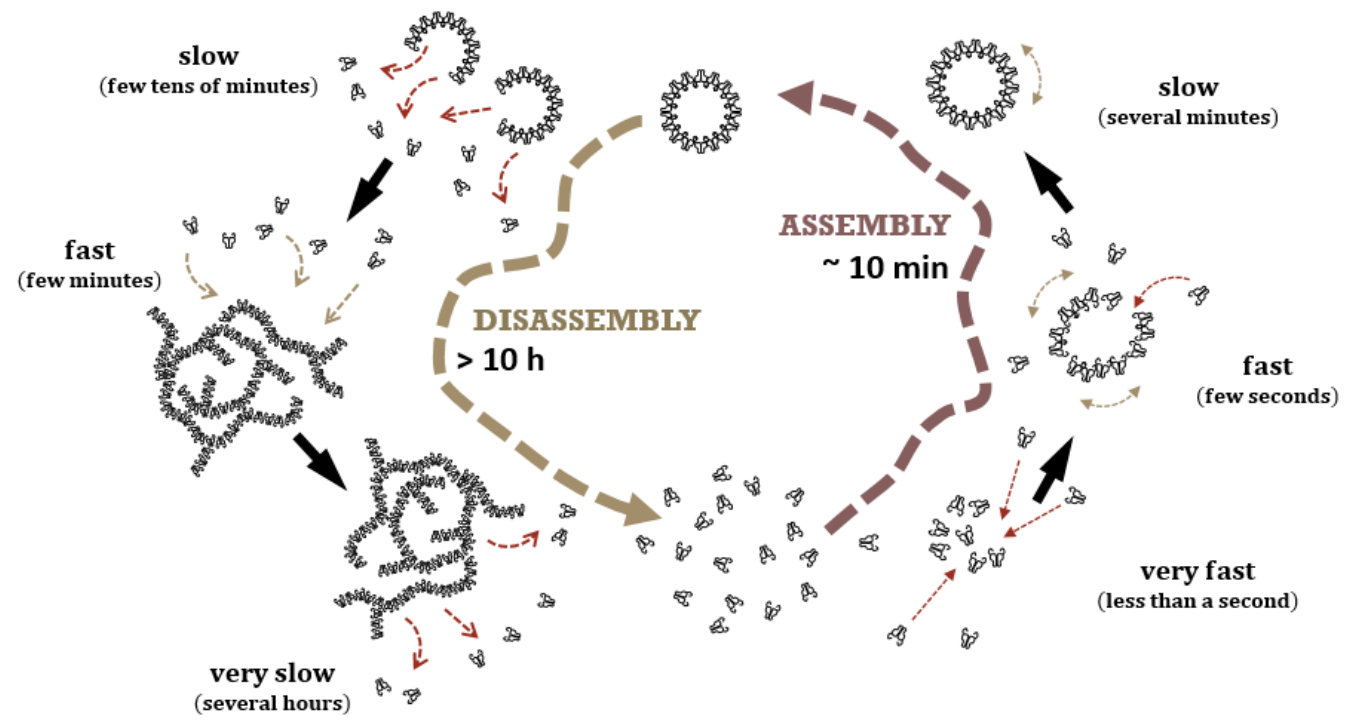

Figure 6: Proposed assembly and disassembly pathways for Cp149 empty capsids. 
Time-resolved studies with fine structural information are increasingly required to better understand the dynamical pathways of viral assembly and disassembly. Elucidating how these pathways are linked to viral replication will hopefully be useful to combat viral infections and to halt outbreaks in their early stage.

\section{Acknowledgement}

The authors thank Mehdi Zeghal, Anja Böckmann, Jéril Degrouard and Didier Law-Hine for fruitful advice. M.C. is supported by the "IDI 2016" project funded by the IDEX Paris-Saclay, ANR-11-IDEX-0003-02. G.T. and S.B. acknowledge financial support from the Agence Nationale de Recherches sur le Sida et les hépatites virales (ANRS). The electron microscopy imaging is supported by "Investissements d'Avenir" LabEx PALM (ANR10-LABX-0039-PALM). We also acknowledge the European Synchrotron Radiation Facility (ESRF) and the synchrotron SOLEIL for allocation of synchrotron beam time on ID02 and SWING beamlines, respectively.

\section{Supporting Information Available}

Size distribution of Cp149 empty capsid, details on mean aggregation number, details on SVD analysis, characteristic timescales, scattering patterns of capsid, subunit and late intermediates, static light scattering and HBV life cycle.

\section{References}

(1) Hiebert, E.; Bancroft, J.; Bracker, C. The assembly in vitro of some small spherical viruses, hybrid viruses, and other nucleoproteins. Virology 1968, 34, 492-508.

(2) Birnbaum, F.; Nassal, M. Hepatitis B virus nucleocapsid assembly: primary structure requirements in the core protein. Journal of virology 1990, $\underline{64}, 3319-3330$. 
(3) Caspar, D. L. D.; Klug, A. Physical Principles in the Construction of Regular Viruses. Cold Spring Harbor Symposia on Quantitative Biology 1962, 27, 1-24.

(4) Zandi, R.; Dragnea, B.; Travesset, A.; Podgornik, R. On virus growth and form. Physics Reports 2020, 847, 1-102.

(5) Baker, B. M.; Murphy, K. P. Methods in Enzymology; Elsevier, 1998; pp 294-315.

(6) Kegel, W. K.; van der Schoot, P. Competing Hydrophobic and Screened-Coulomb Interactions in Hepatitis B Virus Capsid Assembly. Biophysical Journal 2004, 86, 39053913.

(7) Šiber, A.; Podgornik, R. Role of electrostatic interactions in the assembly of empty spherical viral capsids. Physical Review E 2007, $\underline{76}$.

(8) Sun, X.; Li, D.; Wang, Z.; Yin, P.; Hu, R.; Li, H.; Liu, Q.; Gao, Y.; Ren, B.; Zheng, J. et al. Role of Protein Charge Density on Hepatitis B Virus Capsid Formation. ACS Omega 2018, 3, 4384-4391.

(9) Chen, J.; Chevreuil, M.; Combet, S.; Lansac, Y.; Tresset, G. Investigating the thermal dissociation of viral capsid by lattice model. J. Phys. Condens. Matter 2017, 29, 474001.

(10) Tresset, G.; Chen, J.; Chevreuil, M.; Nhiri, N.; Jacquet, E.; Lansac, Y. TwoDimensional Phase Transition of Viral Capsid Gives Insights into Subunit Interactions. Physical Review Applied 2017, $\underline{7}$.

(11) Ceres, P.; Zlotnick, A. Weak Protein-Protein Interactions Are Sufficient To Drive Assembly of Hepatitis B Virus Capsids. Biochemistry 2002, 41, 11525-11531.

(12) Zandi, R.; van der Schoot, P.; Reguera, D.; Kegel, W.; Reiss, H. Classical nucleation theory of virus capsids. Biophys. J. 2006, 90, 1939-1948.

(13) Hagan, M. F. Advances in Chemical Physics; John Wiley \& Sons, Inc., 2014; pp 1-68. 
(14) Zlotnick, A.; Johnson, J. M.; Wingfield, P. W.; Stahl, S. J.; Endres, D. A Theoretical Model Successfully Identifies Features of Hepatitis B Virus Capsid Assembly†. Biochemistry 1999, 38, 14644-14652.

(15) Holmes, K.; Shepherd, D. A.; Ashcroft, A. E.; Whelan, M.; Rowlands, D. J.; Stonehouse, N. J. Assembly Pathway of Hepatitis B Core Virus-like Particles from Genetically Fused Dimers. Journal of Biological Chemistry 2015, 290, 16238-16245.

(16) Pierson, E. E.; Keifer, D. Z.; Selzer, L.; Lee, L. S.; Contino, N. C.; Wang, J. C.-Y.; Zlotnick, A.; Jarrold, M. F. Detection of Late Intermediates in Virus Capsid Assembly by Charge Detection Mass Spectrometry. Journal of the American Chemical Society 2014, 136, 3536-3541.

(17) Katen, S. P.; Chirapu, S. R.; Finn, M. G.; Zlotnick, A. Trapping of Hepatitis B Virus Capsid Assembly Intermediates by Phenylpropenamide Assembly Accelerators. ACS Chemical Biology 2010, $\underline{5}, 1125-1136$.

(18) Kondylis, P.; Schlicksup, C. J.; Katen, S. P.; Lee, L. S.; Zlotnick, A.; Jacobson, S. C. Evolution of Intermediates during Capsid Assembly of Hepatitis B Virus with Phenylpropenamide-Based Antivirals. ACS Infectious Diseases 2019, $\underline{5}$, 769-777.

(19) Singh, S.; Zlotnick, A. Observed Hysteresis of Virus Capsid Disassembly Is Implicit in Kinetic Models of Assembly. Journal of Biological Chemistry 2003, 278, 18249-18255.

(20) Narayanan, T.; Wacklin, H.; Konovalov, O.; Lund, R. Recent applications of synchrotron radiation and neutrons in the study of soft matter. Crystallogr. Rev. 2017, 23, $160-226$.

(21) Henry, E.; Hofrichter, J. Methods in Enzymology; Elsevier, 1992; pp 129-192.

(22) Williamson, T. E.; Craig, B. A.; Kondrashkina, E.; Bailey-Kellogg, C.; Friedman, A. M. 
Analysis of self-associating proteins by singular value decomposition of solution scattering data. Biophysical Journal 2008, 94, 4906-4923.

(23) Berthet-Colominas, C.; Cuillel, M.; Koch, M. H. J.; Vachette, P.; Jacrot, B. Kinetic study of the self-assembly of brome mosaic virus capsid. Eur. Biophys. J. 1987, 15, $159-168$.

(24) Tuma, R.; Tsuruta, H.; French, K. H.; Prevelige, P. E. Detection of intermediates and kinetic control during assembly of bacteriophage P22 procapsid. J. Mol. Biol. 2008, 381, 1395-1406.

(25) Kler, S.; Asor, R.; Li, C.; Ginsburg, A.; Harries, D.; Oppenheim, A.; Zlotnick, A.; Raviv, U. RNA encapsidation by SV40-derived nanoparticles follows a rapid two-state mechanism. J. Am. Chem. Soc. 2012, 134, 8823-8830.

(26) Tresset, G.; Coeur, C. L.; Bryche, J.-F.; Tatou, M.; Zeghal, M.; Charpilienne, A.; Poncet, D.; Constantin, D.; Bressanelli, S. Norovirus Capsid Proteins Self-Assemble through Biphasic Kinetics via Long-Lived Stave-like Intermediates. Journal of the American Chemical Society 2013, 135, 15373-15381.

(27) Law-Hine, D.; Zeghal, M.; Bressanelli, S.; Constantin, D.; Tresset, G. Identification of a major intermediate along the self-assembly pathway of an icosahedral viral capsid by using an analytical model of a spherical patch. Soft Matter 2016, 12, 6728-6736.

(28) Burke, A.; Chevreuil, M.; Paris, A.; de La Grange, V.; Goldmann, C.; Pérez, J.; Constantin, D.; Tresset, G. Nanoparticle-Templated Self-Assembly of Viral Capsids Probed by Time-Resolved Absorbance Spectroscopy and X-Ray Scattering. Phys. Rev. Applied 2018, 10, 054065 .

(29) Chevreuil, M.; Law-Hine, D.; Chen, J.; Bressanelli, S.; Combet, S.; Constantin, D.; Degrouard, J.; Möller, J.; Zeghal, M.; Tresset, G. Nonequilibrium self-assembly 
dynamics of icosahedral viral capsids packaging genome or polyelectrolyte. Nature Communications 2018, 9 .

(30) Law-Hine, D.; Sahoo, A. K.; Bailleux, V.; Zeghal, M.; Prevost, S.; Maiti, P. K.; Bressanelli, S.; Constantin, D.; Tresset, G. Reconstruction of the Disassembly Pathway of an Icosahedral Viral Capsid and Shape Determination of Two Successive Intermediates. The Journal of Physical Chemistry Letters 2015, 6, 3471-3476.

(31) Asor, R.; Khaykelson, D.; Ben-nun Shaul, O.; Levi-Kalisman, Y.; Oppenheim, A.; Raviv, U. pH stability and disassembly mechanism of wild-type simian virus 40 . Soft

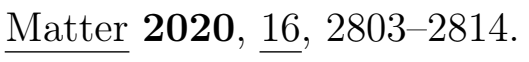

(32) Heger-Stevic, J.; Kolb, P.; Walker, A.; Nassal, M. Methods in Molecular Biology; Springer New York, 2018; pp 503-531.

(33) Porterfield, J. Z.; Dhason, M. S.; Loeb, D. D.; Nassal, M.; Stray, S. J.; Zlotnick, A. Full-Length Hepatitis B Virus Core Protein Packages Viral and Heterologous RNA with

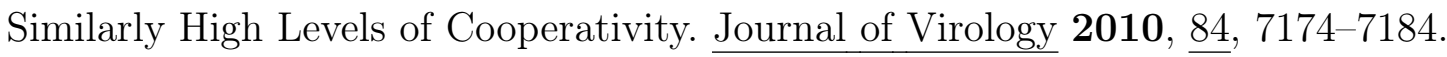

(34) Narayanan, T.; Sztucki, M.; Vaerenbergh, P. V.; Léonardon, J.; Gorini, J.; Claustre, L.; Sever, F.; Morse, J.; Boesecke, P. A multipurpose instrument for time-resolved ultrasmall-angle and coherent X-ray scattering. Journal of Applied Crystallography 2018, 51, 1511-1524.

(35) van der Schoot, P.; Zandi, R. Kinetic theory of virus capsid assembly. Physical Biology 2007, $\underline{4}, 296-304$.

(36) Petoukhov, M. V.; Konarev, P. V.; Kikhney, A. G.; Svergun, D. I. ATSAS 2.1 - towards automated and web-supported small-angle scattering data analysis. Journal of Applied Crystallography 2007, 40, s223-s228. 
(37) Panahandeh, S.; Li, S.; Marichal, L.; Rubim, R. L.; Tresset, G.; Zandi, R. How a Virus Circumvents Energy Barriers to Form Symmetric Shells. ACS Nano 2020, 14, 3170-3180.

(38) Sun, X.; Li, D.; Wang, Z.; Liu, Q.; Wei, Y.; Liu, T. A dimorphism shift of hepatitis B virus capsids in response to ionic conditions. Nanoscale 2018, 10, 16984-16989.

(39) Harms, Z. D.; Selzer, L.; Zlotnick, A.; Jacobson, S. C. Monitoring Assembly of Virus Capsids with Nanofluidic Devices. ACS Nano 2015, 9, 9087-9096.

(40) Asor, R.; Schlicksup, C. J.; Zhao, Z.; Zlotnick, A.; Raviv, U. Rapidly Forming Early Intermediate Structures Dictate the Pathway of Capsid Assembly. Journal of the American Chemical Society 2020, 142, 7868-7882.

(41) Lutomski, C. A.; Lyktey, N. A.; Pierson, E. E.; Zhao, Z.; Zlotnick, A.; Jarrold, M. F. Multiple Pathways in Capsid Assembly. Journal of the American Chemical Society 2018, $140,5784-5790$. 